\title{
Linear and Quadratic PSO Based Color Space Conversion for Sea Target Detection
}

\author{
Saeed mirghasemi, Hadi Sadoghi Yazdi, Mojtaba Lotfizad, Member, IACSIT
}

\begin{abstract}
Sea target detection is an important goal for military purpose and navigation. A new nonlinear quadratic transform is presented to generate target-based color space (TCS) for sea target detection. RGB color space is converted to TCS through a $3 * 3$ matrix, using quadratic transformation. PSO algorithm is used for maximum differentiation between target and non-target clusters which are classified with FCM clustering method. The task of PSO is to minimize the Error value produced by FCM classifier. Experimental results show the ability of the new method in finding sea targets in color images. The superiority of the TCS space to the relative previous works and other color spaces is conspicuous.
\end{abstract}

Index Terms-Quadratic transformation, Color space, Sea target detection, Particle Swarm Optimization, Fuzzy C-Mean.

\section{INTRODUCTION}

Each three-component collection such as $\{$ Red, Green, Blue $\}$ (RGB), and \{Luminance $\mathrm{Y}$, Chrominance $\mathrm{Cr}$, Chrominance $\mathrm{Cb}$ \} ( $\mathrm{YCbCr}$ ) is termed as a color space. Many color spaces are related to each other by linear transformations that are captured by $3 \times 3$ matrices. Hence a given color, and thereby any color image, can be represented in terms of another color space by transforming its $3-\mathrm{D}$ vector representation using the $3 \times 3$ matrix.

The calculations performed in the color space conversion from RGB to $\mathrm{YCbCr}$ are presented below. Parcels of each R, $\mathrm{G}$ and $\mathrm{B}$ input components are considered in the calculation of the output components in the space $\mathrm{YCbCr}$ [1].

$$
\left[\begin{array}{c}
Y \\
C b \\
C r
\end{array}\right]=\left[\begin{array}{ccc}
0.299 & 0.578 & 0.114 \\
-0.169 & -0.331 & 0.5 \\
0.5 & -0.419 & -0.081
\end{array}\right]\left[\begin{array}{l}
R \\
G \\
B
\end{array}\right]
$$

CIE standardized color order systems by specifying the light source, the observer and the methodology used to derive the values for describing color. The XYZ color system was also accepted then and it has been used ever since. In this system, Y represents the brightness (or luminance) of the color, while $\mathrm{X}$ and $\mathrm{Z}$ are virtual (or not physically realizable)

Saeed Mirghasemi is with the Computer \& IT Engineering Department, Islamic Azad Univercity of Parand, IRAN (e-mail: s.mirghasemi@gmail.com) Hadi Sadoghi Yazdi is with the Computer Engineering Department, Ferdowsi University of Mashhad, IRAN (e-mail: h-sadoghi@um.ac.ir)

Mojtaba Lotfizad is with the Electical \& Computer Engineering Department, Tarbiat Modares University of Tehran, IRAN (e-mail:

lotfizad@modares.ac.ir) components of the primary spectra [2]. Many color spaces are presented but other viewpoints are theories of color vision which are derived from the sums and differences of the three cone types. One mechanism (often referred to as the "luminance" mechanism) signals a weighted sum of long-wavelength-selective (L) and medium-wavelength-selective (M) cones, i.e., " $\mathrm{L}+\mathrm{M}$ " (with some debate regarding the contribution of short wavelength selective (S) cones: [3]-[5]. Another viewpoint to color space is the selection of the best space. In this category, different transformations of RGB color space (as HSI, HSV and Lab) were compared to find the best method for separating target/clutter or foreground/background and so on in color images taken by a digital photo camera [6]. Some researchers used a color space in a specific application in the computer vision as the skin detection in HSV [7], face recognition in new space based on K-L1 transform [8], lip tracking into the CIELAB, CIELUV color spaces [9], object recognition into illumination invariant type of RGB color space [10], Image restoration into the CIELAB color space [11], and so on. In [12], the properties of six color spaces are discussed to detect of specific surface defects. They show that these defects are well detected when the clustering analysis is performed in the RGB space. Authors of [13] apply two classification methods using different color spaces. By means of a visual appreciation of the results, they also conclude that the RGB space is the best among all the considered color spaces.

The main target of this paper is to create quadratic transform of the color space with fuzzy c-means criteria. More specific we are going to acquire a $3 * 3$ conversion matrix based on quadratic transformation through PSO search algorithm with FCM clustering criteria. The following sections are a brief survey on sea target detection, introducing our quadratic conversion, using PSO search algorithm to find the optimum conversion matrix and to show the obtained results from different points of views.

\section{A. Target Detection in Sea}

There are not many researches about target detection in sea through direct image processing and among them papers that utilize color features are even fewer. For this reason almost all of the existing approaches suffer from one or more drawbacks like sensitivity to different sensors [14] or invalidity for low SNR images [15]. Many papers have worked on ship detection based on $\mathrm{SAR}^{2}$ images [16-19]. [16]

\footnotetext{
${ }^{1}$ Karhunen-Lo`eve

${ }^{2}$ Synthetic Aperture Radar
} 
presents a new method based on combinatorial improved $\mathrm{PNN}^{3}$ model for ship detection in SAR imagery and [17] proposes a method to reduce speckle noise for SAR images and to improve the detected ratio for SAR ship targets from the SAR imaging mechanism. A specific technique for automatic spot detection, based on the Wavelet Transform is presented and justified in [18]. Marivi et al [19] proposed a ship target detection method of SAR images by means of the discrete wavelet transform, taking advantage of the difference of statistical behavior of the vessels and the sea. All above methods are dependent on SAR images; they expend largely and can only obtain target points, which cannot be used to recognize targets. There are also some papers that use remote sensing images for ship detection [20]. They present a method based on cumulative projection curve (CPC) to estimate the number of ships of small size, which is only efficient on especial images from stationary ships along coastline in a harbor. One of the few researches that uses color feature, from Lab color coordinate system, for sea target detection is [21]. They presented a definition on the degree of overlap between two clusters and developed an algorithm for calculating the overlap rate. Using this theory, they also developed a new hierarchical cluster merging algorithm for image segmentation and applied it to the ship detection in high resolution images. [22] is one of the several papers that worked on IR images. They used PCA, Bayes classification and wavelet-denoising to classify the sea targets, but in several papers, limitations and disadvantages of the methods based on statistical analysis are pointed out [24]. One of the papers that have more superiority to previous works in visible images domain is [23]. Their work is based on calculating different chaos by obtaining largest Lyapunov exponent of target and sea background which is not appropriate for images that contain seaside or some low chaos objects. Also the authors have proposed [24] based on the natural measure feature. Although the results of the method are considerable for some images but it still suffers from previously mentioned imperfection and needs analyzing several frames for exact results. So it is still important to find new methods of detecting target from sea background. Except [21] all above researches have worked on grey level images and for sure our method is one of the few one that utilizes color feature for sea target detection.

\section{B. Motivation}

The aforementioned works on target detection in sea appear to justify the implementation of the new color space to find targets in the sea. From our previous experiments [25-27] we know that linear transformation cannot satisfy clustering criteria in target detection domain. So in this paper quadratic transformation is introduced which is a new work in creating the new color space. Creating an optimum connection between one of the most common clustering methods which is the FCM classifier, and the PSO search method is another motivation. All these points encourage us to present the target-based color space (TCS) for suitable sea target

\footnotetext{
${ }^{3}$ Probabilistic Neural Network
}

detection. This paper is organized as follows. Section II provides information about the details of creating the new color space. Subsections A and B are about linear and quadratic transformations and their relationship to the FCM and PSO. In subsection $\mathrm{C}$ we described the PSO algorithm and its implementation for purchasing our goals. Section III is dedicated to experimental results and the new color space is tested from different points of view. Section IV is about conclusions and a review of the general PSO algorithm is presented in appendix.

\section{THE PROPOSED APPROACH}

\section{A. Linear Transformation}

In [25]-[27] we proposed a PSO based method for skin, glider and lip detection respectively, where they used linear transform and Hard C-mean (HCM) clustering method for classification. In this paper we are going to extend this method with FCM rather than HCM. In this way, first we introduce the same linear transformation from original space to the new one which is performs by the following equation:

$$
y=x * w
$$

where $x$ is a point in the original space $(1 \times 3$ vector containing $\mathrm{R}, \mathrm{G}$ and $\mathrm{B}$ information), $y$ is the corresponding point in the new space, and $W$ is a $3 * 3$ transformation matrix. Then we use the FCM clustering method in the data achieved in the new space which is based on the minimization of the following objective function:

$$
\begin{array}{r}
J_{m}(U, V)=\sum_{k=1}^{n} \sum_{i=1}^{c} u_{i k}^{m}\left\|y_{k}-v_{i}\right\|^{2}, \\
J_{m}(U, V)=\sum_{k=1}^{n} \sum_{i=1}^{c} u_{i k}^{m}\left\|(x * \mathrm{~W})_{k}-v_{i}\right\|^{2},
\end{array}
$$

where $Y=\left\{y_{1}, y_{2}, \ldots, y_{n}\right\}$ is a finite subset of an s-dimensional vector space over the real data. $y$ denotes the set of feature vectors. $c$ is the number of clusters, and $m>1$ is the fuzziness index. The matrix $U=\left[u_{i k}\right]_{c \times n}$ is called a constrained fuzzy c partition of $y$ if the entries of $U$ satisfy,

$$
\begin{aligned}
& u_{i k} \in[0,1], \quad i=1,2, \ldots, c \quad k=1,2, \ldots, n \\
& \sum_{i=1}^{c} u_{i k}=1, \quad k=1,2, \ldots, n \\
& 0<\sum_{k=1}^{n} u_{i k}<n, \quad i=1,2, \ldots, c .
\end{aligned}
$$

And $u_{i k}$ is the degree of membership of $k$-th data to $i$-th clusters. $V=\left\{v_{1}, v_{2}, \ldots, v_{c}\right\}$ is the cluster prototypes set. $v_{i} \in R^{s}$ is the center of the $i$-th cluster.

In order to minimize the clustering error and obtain the optimum transformation weights, the PSO search method is utilized. In fact, PSO searches a 9-dimensional space for optimum weights and results in an optimum clustering $3 * 3$ $W$. More details about operation of our method are discussed 
in introducing Quadratic transform.

\section{B. Quadratic Transformation}

Although previous obtained $W$ is powerful in clustering domain but we are going to show there is a better approach for finding weights in a way that results in better clustering especially in images that have complicated back grounds or images that targets and non-targets have the same color contents. In this way, first we convert the training sample images from the RGB color space to a new space through a quadratic transformation which is performed by the following equation:

$$
y=0.5 *\left(W *(x * W)^{T}\right)^{T}+x * W
$$

The training samples are extracted from target and non-target pixels of the original image. Again the FCM method is used to cluster the obtained data in the new space. Therefore we have to minimize the mentioned objective function in (3) with a different $y$ that is changed and is obtained from (4). By rewriting the objective function, we are having the following equation:

$$
J_{m}(U, V)=\sum_{k=1}^{n} \sum_{i=1}^{c} u_{i k}^{m}\left\|\left(0.5 *\left(\mathrm{~W} *(\mathrm{x} * \mathrm{~W})^{\mathrm{T}}\right)^{\mathrm{T}}+\mathrm{x} * \mathrm{~W}\right)_{k}-v_{i}\right\|^{2}
$$

The common solution for solving this non-linear problem is through Lagrange multipliers and for optimizing the final solution it is sufficient to solve the following set of equations:

$$
\left\{\begin{array}{l}
\frac{\partial J(U, V)}{\partial W}=0 \\
\frac{\partial \ell(U, V)}{\partial X}=0 \\
\frac{\partial \ell(U, V)}{\partial v}=0
\end{array}\right.
$$

It is obvious that by expanding (7), because of the power of 2 , we are going to have a non-linear equation with powers of 4 and 3 . So the new objective function is non-convex and it is hard to be solved through Lagrange multipliers. In fact the prior equation because of its non-quadratic structure does not seem to have a routine solution. So we use the PSO approach to solve this non-linear non-convex problem.

There for, our new work has two substantial superiorities into our previous works and related mentioned references:

a) Our previous works, [25] and [26] used hard c-mean for clustering and [27] used Euclidean distance for differentiation between target and non-target. There for, for the first time we have used combination of weighted FCM and the PSO for optimum clustering.

b) All mentioned references used linear transformation for representing the new color space. Presenting quadratic transformation for creating the new color space is our original solution to solve some real-world clustering problems.

\section{Finding optimized conversion matrix using PSO algorithm}

For a quick review of the PSO algorithm see appendix (section 5). Our major goal in finding conversion matrix is to differentiate between target and non-target pixels as much as possible. For this reason in our method we have the target and non-target pixels which are used for training. After choosing pieces of target and non-target pixels, we put them along each other as a training sample. As mentioned $W$ is a $3 * 3$ matrix, after transforming these training pixels to the new space with $W$, we use the FCM method for clustering. Then we define a function to calculate the error value of FCM clustering. More clearly we need a function for tuning the velocity and position of our particles in the PSO space. This function is a criterion that connects FCM and PSO in a way that results in a more differentiator space between target and non-target pixels. We call this criteria and function, Error value and Cost function respectively and we want Error value as minimum as possible. Fig. 1 shows the flowchart of the proposed algorithm.

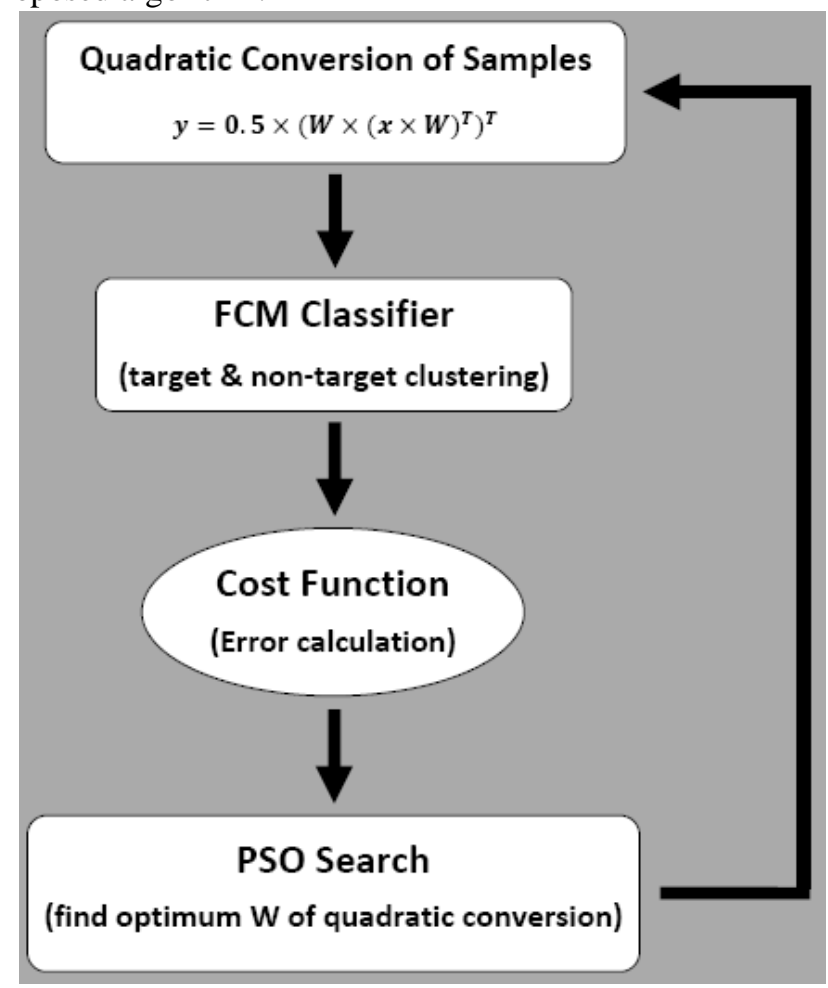

Fig.1 Different level flowchart of creating the new color space

The following pseudo code describes our method in finding the best clustering conversion matrix.

Comment: PSO-based optimizing conversion matrix problem algorithm

Comment: Define the solution space, fitness function, and population size

For each $W$,

\section{Initialize:}

Minimum and maximum value of particles velocity in each dimension $\left[v_{\min } v_{\max }\right]$,

Maximum iteration, number of particles,

Minimum and maximum value of particles value in each dimension $\left[x_{\min } x_{\max }\right]$,

Initial and final value of Inertia weighting factor $w$,

Determine initial value for particles $\boldsymbol{x}$. 
While Iteration <Max iteration or population uniformity $<$ threshold

Update inertia weighting factor $w$

Call conversion and error calculating function; Comment : Cost function

Finding pbest and gbest;

Comment: pbest and gbest are best position for each and all particles respectively,

Update $\overrightarrow{\boldsymbol{v}}_{\boldsymbol{i}}^{\boldsymbol{k}+1}$ and $\overrightarrow{\boldsymbol{x}}_{\boldsymbol{i}}^{\boldsymbol{k}+1}$; Comment: According to (11),

\section{End While}

\section{End For}

Display founded best $\overrightarrow{\boldsymbol{x}}$

This structure includes three main parts:

a) The PSO algorithm.

b) Cost function in the PSO algorithm for Finding optimized conversion matrix.

c) Velocity weight update control.

1) Using the PSO algorithm for solving the problem: The PSO algorithm has been described in previous section and here we explain matching it to this problem. $\vec{x}_{i}=\left(x_{i 1}, x_{i 2}, \ldots, x_{i n}\right)$ is the $\mathrm{i}^{\text {th }}$ particle with $\boldsymbol{n}$ dimensions. Here $W$ has 9 members so here, search space is 9-D $(n=9) \quad$ and $\vec{x}_{i}=\left(x_{i 1}, x_{i 2}, \ldots, x_{i 9}\right)$ i.e. each particle proposes one variable with 9 dimensions. Cost function determines the value of each particle and search is done based on the cost of all particles as explained in section $\mathrm{B}$ Next sub-section includes the PSO cost function description in finding the optimized $W$.

2) Cost function in the PSO algorithm: In the cost function, after linear/quadratic conversion of image to the new color space through the proposed $W$ by PSO (for each R,G,B component of the new image), the Error value is getting calculated through Cost function and the maximum value of the Error value is fed back to the PSO. It means that we are going to minimize the worst obtained error value.

3) Error value: In our algorithm the Error value is obtained by Cost function in which, by means of FCM method we are trying to produce an error based on belonging of the transformed training pixels into target and non-target clusters. For this sake, we divide the transformed pixels into two groups and try to count the number of pixels that are not in their own clusters. It is obvious that the more the number of training sample pixels are the more the real Error value is expectable, and the lower the Error value, the better the clustering is performed. So during the PSO execution the aim is to minimize the Error value which is described as follow:

$$
\text { Error valu e }=\left(e_{n 1}+e_{n 2}\right)
$$

where $\mathrm{e}_{\mathrm{n} 1}$ denotes the number of pixels that do not belong to the first cluster and $\mathrm{e}_{\mathrm{n} 2}$ denotes the number of pixels that not belonng to the second cluster.
4) Velocity weight update control: This operation is getting done with a factor called inertia weight. Many references pointed that good values for inertia weight are usually slightly less than 1. From our experiments in PSO particle velocity control we understand that choosing the inertia weight between 0.5 and 1 result in maximum velocity coincidence. Then in the standard PSO, the inertia weight is introduced as a decreasing function which is set to a higher value $w_{\text {initialize }}=1$ and finally it becomes $w_{\text {final }}=0.5$. Linear relation is defined per iteration according to following,

$w=\left(w_{\text {initialize }}-w_{\text {final }}\right) \frac{\left(k_{\max }-k\right)}{k_{\max }}+w_{\text {final }}$

where $w_{\text {initialize }}$ initializes the value of the weight factor and the final weight is $w_{\text {final }}, k$ is the iteration number and $k_{\max }$ is maximum iteration. In the first iteration $k$ is equated to zero and $w$ is $w_{\text {initialize }}$ and gradually decreases to $w_{\text {final }}$. Choosing values out of this interval lead to uncontrollable particle velocity which affects the best global solution.

\section{Linear and Quadratic Transform Complexity Comparison in Real Time Application}

It is obvious from (6) that the quadratic transform has 3 more multiply, one more summation and two more matrixes transpose in contrast with the linear one. Because this quadratic conversion repeats in every PSO iteration, the total additional tasks that should be done multiply in PSO maximum iteration number which is a considerable amount of operations. For real time application, we should use a proper software like $\mathrm{C}++$ which may take substantial time to do these additional operations.

For applying the whole approach in real time applications, we should say, after obtaining the final conversion matrix $(W)$, the linear and the quadratic transforms take almost the same time to convert the image to the new color space. The consuming time is small enough to be applied in real time applications. But since, running the PSO code take more time to be completed, applying the whole method for real time applications is not appropriate.

\section{EXPERIMENTAL REULTS}

Because of the security problems sea target databases, unlike face and skin images, are not published. Lack of famous color images for sea target detection, forced us to create our own database. We have successfully tested our method on 3 different databases. First database is about 2000 frames of the sea targets from several mainstreams movies.

The second database is collected from different military websites and the third one is database from low quality movies filmed by one of famous military companies for sea target detection purposes. It is obvious that because of observing framing rules the third database has the best result.

In this section, we are going to exhibit the functionality of 
our method in different conditions and different applications. First of all, we'll show how this method works. Comparing linear and quadratic transformations is the next step. Ability of our method in segmenting several targets, working on untrained data and robustness of new color space on complicated background images are also illustrated.

If we use learning samples which is a $25 * 50$ image and is shown in Fig. 2-a, the following conversion matrix is obtained:

\section{$1.5387 \quad 0.0053 \quad 2.2600$ \\ $2.1142 \quad 0.1594 \quad 2.9901$ \\ $2.9068 \quad 1.9066 \quad 2.3567$}

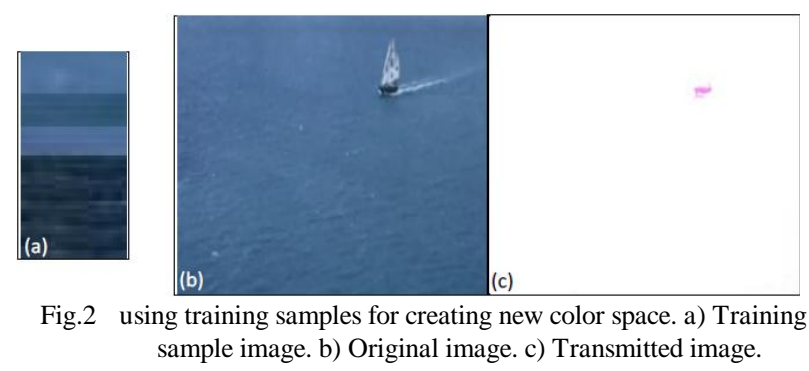

The first half of the training sample image contains non-target pixels and the other half contains target pixels. Fig. 2-b and 2-c shows the original image and the converted image respectively. After the PSO search stage with 50 particles and 100 iteration levels, the minimum error of 0 is obtained.

In contrast with linear transformation, the quadratic one has two considerable superiorities:

a. The quadratic conversion is resulted in minimum Error value after fewer iteration steps. It means that not only more minimum error value is reachable through quadratic conversion, but also less iteration number is required.

b. It is more efficient in critical images that have the same chrome contents in target and non-target pixels. For example images that have sky or beach on them.

Fig. 3 shows obtained results for two of these critical images. For first image (Fig. 3-a), linear transmission the error value is 31 , while for the quadratic one it is 0 . Besides quadratic transform is converged to 0 Error value after 8 iterations while the linear one couldn't reach better than 31 Error value with maximum iteration of 90 and 50 particles. Fig. 3-e, f also show the result for linear and quadratic conversion of Fig. 3-d. Although the obtained Error value for both converted images are 0 , but the quadratic one reaches to this error value after 6 iterations while the linear reaches after 10 iterations.

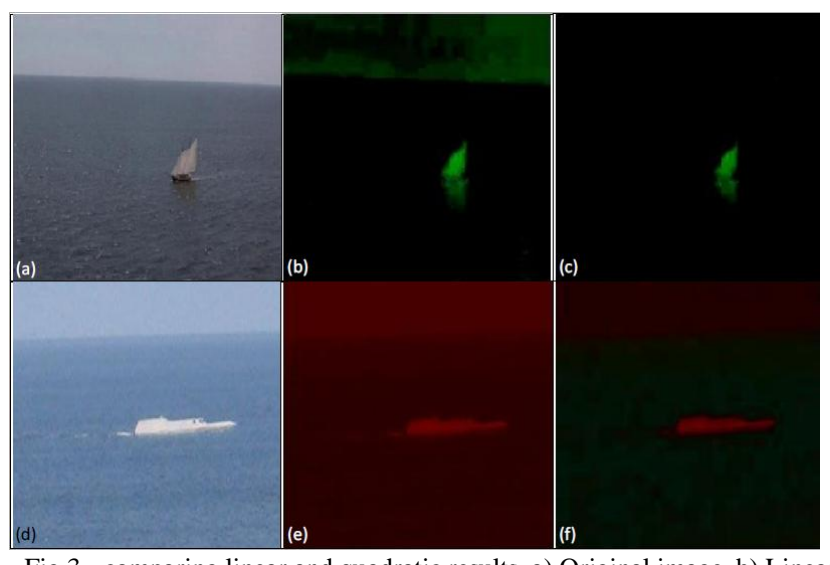

Fig.3 comparing linear and quadratic results. a) Original image. b) Linear conversion result. c) Quadratic conversion result.

One of the important advantages of this new color space is its capability with different situations which is shown in Fig. 4. Fig. 4-b shows the result with error value of 4 and every 8 targets are detected while Fig. 4-c can extract 6 targets with error value of 5 . This is because the number of maximum iteration and particles in the second result decreased and it can be vary based on user demand for different purposes.

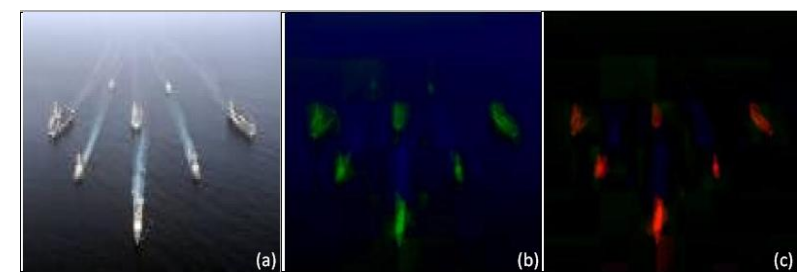

Fig.4 several target detection. a) Original image. b) Complete detection. c) Incomplete detection.

For expressing the capability of this method we used the conversion matrix obtained by the training sample of Fig. 4 and have shown the result in Fig. 5. This means that our method is able to detect targets that are not even used for training. For this test maximum iteration, particle numbers and error value are 90, 40 and 0 respectively.

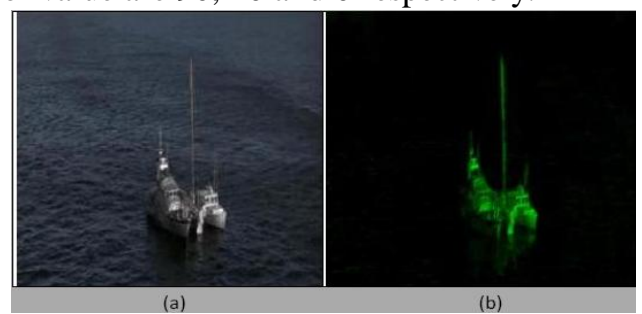

Fig.5 Test of new color space on image that doesn't have been seen before a) Original image. b) Extracted result.

As an application for this feature we can use our new method in defense industries for target destruction on scenes that we have information about. In this way the target is being marked by a pilot from a warplane or a sailor from a warship which means a window is being selected that has target and non-target on it. Then the image is getting processed by our new filter and if the result is good enough, it is getting confirmed by the user for firing the missile, torpedo or so on.

Utilizing color features in our method made it possible to have good result even in images that have a complicated background. For this reason a sample image that contains non-targets moreover sea (like sky, seaside and etc) and the 
converted image are shown in Fig. 6. This example demonstrates the capability of our method in detecting target even when the frame is not marked properly.

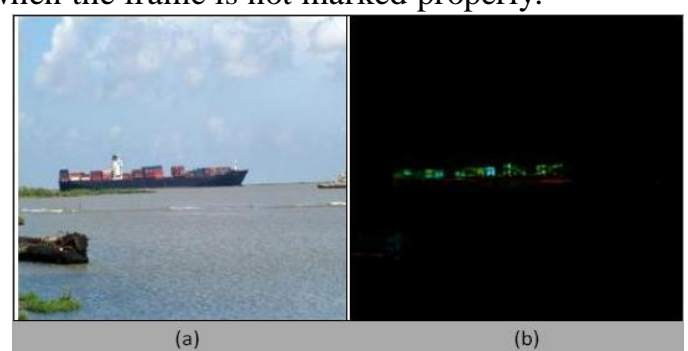

Fig.6 efficiency of our new method in complicated background pictures. a) Original picture. b) Converted image.

We should say that in images that target and non-target have the same color container; our method can have unsuitable results. One of these images is shown in Fig. 7. For not having such consequences especially in military applications, the frame could be selected more intelligently with guidance of the user that knows the approximate location of the target.

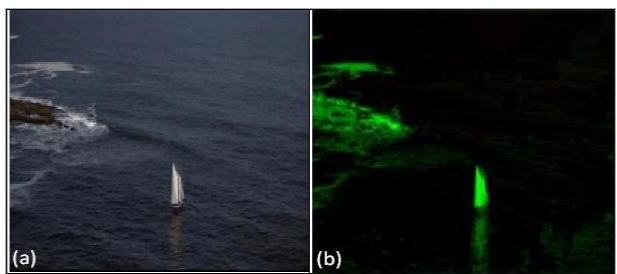

Fig.7 inappropriate result in target detection. a) Original image. b) Result in new color space.

In special databases like our third database, which were particularly gathered for sea target detection purposes, we can use one conversion matrix in most of the pictures for target detection. For example in Fig. 8 the following matrix is obtained from Fig. 8-a with 19 particles and 80 iteration levels:$$
-1.3405 \quad-0.4130 \quad 0.0005
$$$$
0.3790 \quad 0.2111 \quad 3.2416
$$$$
2.8010 \quad 0.3634 \quad 2.6539
$$

As depicted in Fig. 8 the above matrix has the same effect in target and non-target clustering in other images. Although lighting, target and back ground conditions are somehow different, but the target is segmented in all images, using the same conversion matrix.

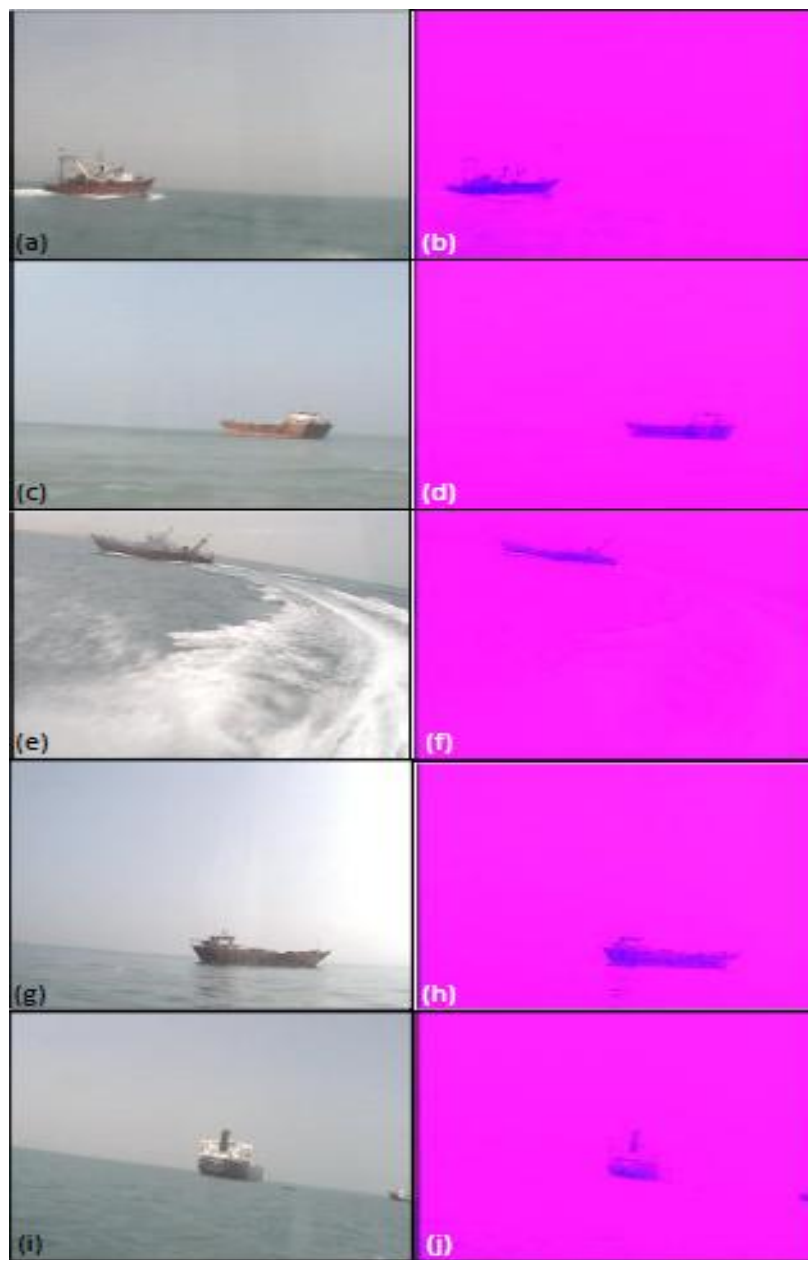

Fig.8 using one conversion matrix in a special database for target detection. a) The image that the conversion matrix is extracted from. $b-j$ ) The effect of the previous conversion matrix in other images from the same database.

At last we are going to compare our new color space in contrast with some classic color spaces. Therefor an image in different spaces is shown in Fig. 9 to demonstrate the robustness of the new color space.
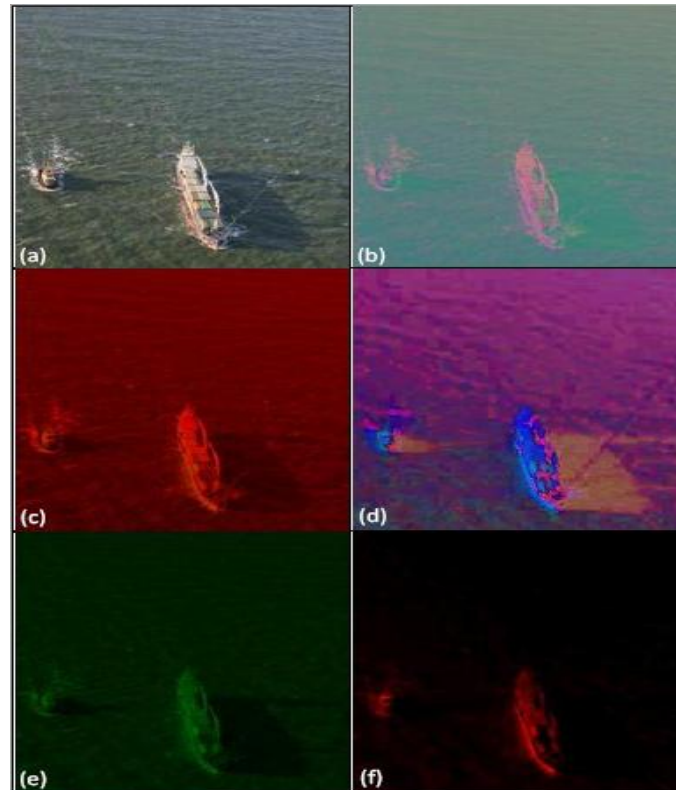

Fig.9 new color space in contrast with some classic color spaces. a) Original image. b) YCbCr color space. c) YIQ color space. d) HSV color space. e) Linear conversion. f) Quadratic conversion. 


\section{CONCLUSIONS}

Conventional clustering algorithms fail to solve some real-world clustering problems. A PSO-adjusted space conversion based on quadratic conversion is developed to deal with a particular kind of clustering problems which is target detection in sea pictures. In the newly generated space, patterns (points) are rearranged in such a way that FCM can recognize intrinsic grouping of the given data. There are three innovations in this paper. First we utilized a novel combination of FCM clustering and PSO search methods. Introducing quadratic color space conversion is the second. And thirdly we successfully tested this new color space for target detection in sea pictures. Experimental results show that not only quadratic conversion is a new criterion for creating a more differentiator color space, but also the application in the sea target detection is a new and successful experience. All above abilities made the new color space to be efficient in more general frames. It means that our new method, unlike the related mentioned work in section I that are efficient in special kind of images or frames with special angle of view, is applicable in almost all color images and frames. For sure our work is one of the first research works on sea target detection that utilizes color features. One of our future works is to generalize our method to any other benchmark problems. In general such a space conversion that can result in the discovery of natural grouping in data would be appreciable. Because, it is valuable in many areas like: signal representation, pattern classification/recognition, pattern understanding/analysis, and data mining. In other words, it makes it possible to work in a more sensible and more desirable space.

\section{APPENDIX}

Particle swarm optimization (PSO) is a swarm intelligence based algorithm to find a solution to an optimization problem in a search space, or model and predict social behavior in the presence of objectives. The particle swarm optimization algorithm was first described in 1995 by James Kennedy and Russell C. Eberhart [28],[29]. The techniques have evolved greatly since then, and the original version of the algorithm is barely recognizable in the current ones. The swarm is typically modeled by particles in multidimensional space that have a position and a velocity. These particles fly through hyperspace (i.e., $R^{n}$ ) and have two essential reasoning capabilities: their memory of their own best position (pbest) and knowledge of the global or their neighborhood's best (gbest). In a minimization optimization problem, problems are formulated so that "best" simply means the position with the smallest objective value. Members of a swarm communicate good positions to each other and adjust their own position and velocity based on these good positions. So a particle has the following information to make a suitable change in its position and velocity:

- A global best that is known to all and immediately updated when a new best position is found by any particle in the swarm.
- Neighborhood best that the particle obtains by communicating with a subset of the swarm.

- The local best, which is the best solution that the particle has seen.

In this work, the global variant of the PSO was considered because it exhibited faster convergence compared to the local one [30]. Suppose that the search space is D-dimensional, then the $\mathrm{i}^{\text {th }}$ particle of the swarm can be represented by a D-dimensional vector, $\mathrm{X}_{\mathrm{i}}=\left(\mathrm{x}_{\mathrm{i} 1}, \mathrm{x}_{\mathrm{i} 2} \ldots \mathrm{x}_{\mathrm{iD}}\right)$. The velocity (position change) of this particle, can be represented by another D-dimensional vector $\mathrm{V}_{\mathrm{i}}=\left(\mathrm{v}_{\mathrm{i} 1}, \mathrm{v}_{\mathrm{i} 2} \ldots \mathrm{v}_{\mathrm{iD}}\right)$. The Use of inertia weight $\mathrm{w}$ has been proved to result in improved performance [31]. In a PSO system based on inertia weight update of particles is accomplished by the following equations:

$$
\mathrm{v}_{\mathrm{id}}^{\mathrm{k}+1}=\mathrm{Wv}_{\mathrm{id}}^{\mathrm{k}}+\mathrm{c} 1 \times \mathrm{r} 1\left(\mathrm{xpbest}_{\mathrm{id}}-\mathrm{x}_{\mathrm{id}}\right)+\mathrm{c} 2 \times \mathrm{r} 2\left(\mathrm{xgbest}_{\mathrm{id}}-\mathrm{x}_{\mathrm{id}}\right)
$$

$$
\mathrm{x}_{\mathrm{id}}^{\mathrm{k}+1}=\mathrm{X}_{\mathrm{id}}^{\mathrm{k}}+\mathrm{v}_{\mathrm{id}}^{\mathrm{k}+1}
$$

where $\mathrm{d}=1,2, \ldots, \mathrm{D} ; \mathrm{i}=1,2, \ldots, \mathrm{N}$, and $\mathrm{N}$ is the size of the swarm; $\mathrm{c} 1$ and $\mathrm{c} 2$ are positive constants; $\mathrm{r} 1$ and $\mathrm{r} 2$ are random numbers, uniformly distributed in $[0,1]$; and $\mathrm{k}=1$, $2, \ldots$, denotes the iteration number.

\section{REFERENCES}

[1] Bhaskaran V, Konstantinides K. Image and video compression standards algorithms and architectures. USA: Kluwer Academic Publishers; 1999.

[2] Wyszecki, G., Stiles, W.S., 1982. Color Science: Concepts and Methods, Quantitative Data and Formulae, Second Edition Wiley, New York.

[3] A. Eisner, D. I. A. MacLeod "Blue Sensitive Cones do not Contribute to Luminance," Journal of the Optical Society of America, vol. 70, pp.121-123, 1980.

[4] R. M. Boynton, R. T. Eskew, C. X. Olson, "Blue Cones Contribute to Border Distinctness," Vision Research, vol.25, no.9, 1349-1352, 1985.

[5] A. Stockman, D. I. A. MacLeod, D. D. DePriest, "The Temporal Properties of the Human Short-Wave Photoreceptors and Their Associated Pathways," Vision Research, vol.31, 189-209, 1991.

[6] I. Philipp, T. Rath, "Improving Plant Discrimination in Image Processing by Use of Different Color Space Transformations," Computers and Electronics in Agriculture, vol.35, pp.1-15, 2002.

[7] L. Sigal, S. Sclaroff, V. Athitsos, "Skin Color-Based Video Segmentation under Time-Varying Illumination," IEEE Trans. on Pattern Analysis and Machine Intelligence, vol. 26, no.7, pp.862-877, July 2004.

[8] C. F. Jones, A. L. Abbott, "Optimization of Color Conversion for Face Recognition," Eurasip Journal on Applied Signal Processing, vol.4, pp.522-529, 2004.

[9] A. W-C. Liew, S.H. Leung, W. H. Lau, "Segmentation of Color Lip Images by Spatial Fuzzy Clustering," IEEE Trans. on Fuzzy System, vol.11, no.4, pp.542-549, Aug. 2003.

[10] A. Diplaros, T. Gevers, I. Patras, "Combining Color and Shape Information for Illumination-Viewpoint Invariant Object Recognition," IEEE Trans. on Image Processing, vol.15, no. 1, pp.1-11, Jan. 2006.

[11] L. Jin, D. Li, "A Switching Vector Median filter Based on the CIELAB Color Space for Color Image Restoration," Signal Processing, vol.87, pp.1345-1354, 2007.

[12] C.C. Brunner, A.G. Maristany, D.A. Butler, D. Vanleuween, J.W. Funck, "An Evaluation of Color Spaces for Detecting Defects in Douglas-.r veneer, Ind. Metrol. 2 (3 and 4) 169-184, 1992.

[13] E. Littmann, H. Ritter, "Adaptive Color Segmentation. A Comparison of Neural and Statistical Methods," IEEE Trans. Neural Networks 8 (1) 175-185, 1997.

[14] Cao Y L, Yang J Y and Ren M G, "Novel object detection method by probability velocity field", Machine Vision Applications in Industrial Inspection VIII, pp. 309-314, 2000.

[15] Su D, Zhang Q H and Xie S H, "Segmentation Algorithm for Multi-targets in Sea Surface", Computer Engineering and Applications, 16, pp. 12-14, 2006. 
[16] Zhenhong Du, Renyi Liu, Nan Liu, Peng Chen, "A New Method for Ship Detection in SAR Imagery based on Combinatorial PNN model", IEEE First Int. Conf. on Intelligent Networks and Intelligent Systems, 2008.

[17] Shi-qi Huang, Dai-zhiLiu, Gui-qingGao, Xi-jianGuo, "A novel method for speckle noise reduction and ship target detection in SAR images", Science Direct, Pattern Recognition, 42(2009)1533-1542, 2009.

[18] Marivi Tello, Carlos Lopez-Martinez, Jordi J. Mallorqui, Ramon Bonastre, "Automatic Detection of Spots and Extraction of Frontiers in SAR Images by Means of the Wavelet Transform: Application to Ship and Coastline Detection", IEEE Int. Con. on Geoscience and Remote Sensing Symposium, IGARSS 2006.

[19] T. Marivi, L. M. Carlos, and J. M. Jordi, "A novel algorithm for ship detection in SAR imagery based on the wavelet transform", IEEE Geoscience And Remote Sensing Letters, vol. 2, pp. 201-205, 2005.

[20] Yonggang Hu, Yi Wu, "Number Estimation of Small-Sized Ships in Remote Sensing Image Based on Cumulative Projection Curve", IEEE Int. Conf. on Audio, Language and Image Processing, (ICALIP),2008.

[21] Zhiling Hong, Qingshan Jiang, Heshan Guan and Fangfei Weng, "Measuring overlap-rate in hierarchical cluster merging for image segmentation and ship detection", Fourth Int. Conf. on Fuzzy Systems and Knowledge Discovery (FSKD), 2007.

[22] Yusuf Yaslan, Bilge Giinsel, "Detection of Sea Targets from Thermal Images", IEEE Signal Processing and Communications Applications Conference, 2004.

[23] Shaoqing Yang, Sihua He, Hongwen Lin, "Video Image Targets Detection Based on the Largest Lyapunov Exponent", IEEE The 9th Int. Conf. for Young Computer Scientists, 2008.

[24] Sihua He, Shaoqing Yang, Aiguo Shi, Tianwei Li, "A Novel Image Moving Sea Targets Detection Method Based on Natural Measure Feature", IEEE Int. Symposium on Information Science and Engieering, 2008.

[25] M. Alipoor, Hadi Sadoghi Yazdi, "A Novel Clustering Method Using PSO-Adjusted Space Conversion", ISFS 2008.

[26] Hadi Sadoghi Yazdi, "Target Detection in Scene Utilizing Intelligent Color Space Conversion", $12^{\text {Th }}$ Int. CSI Computer Conf. (CSICC'07), 2006.

[27] Seyyed Meysam Hosseini , Hasan Farsi , Hadi Sadoghi Yazdi, "Best Clustering Around the Color Images", Int. Journal of Computer and Electrical Engineering, Vol. 1, No. 1, April 2009.

[28] R. C. Eberhart and J. Kennedy, "A new optimizer using particle swarm theory", Proc. Int. on Micro Machine and Human science. Japan, pp. 39-43, 1995.

[29] J. Kennedy and R. C. Eberhart, "Particle swarm optimization", Proc. IEEE Int. conf. on Neural Networks. Piscataway, NJ, pp. 1942-1948, 1995.

[30] M. M. Kandil et al., "A New Approach for Optimizing Backpropagation Training with Variable Gain Using PSO", GVIP 05 Conf., CICC, Cairo, Egypt, 2005.

[31] Y. Shi and R. C. Eberhart, "A modified particle swarm optimizer", Proc. IEEE Int. Conf. on Evolutionary Computation. Piscataway, NJ, pp. 69-73, 1998.

Saeed Mirghasemi was born in Tehran, Iran, in1981. He received the B.S. degree in electrical engineering from Semnan University of Iran in 2006, and he is about to receive his M.S. degrees in electrical engineering from Malek Ashtar University of Tehran, Iran. His research interests include adaptive filtering, intelligent image processing and fuzzy processing.

E-mail: s.mirghasemi@gmail.com

Hadi Sadoghi Yazdi was born in Sabzevar, Iran , in 1971. He received the B.S degree in electrical engineering from Ferdowsi University of Mashad in 1994, and then he received to the M.S. and $\mathrm{PhD}$ degrees in electrical engineering from Tarbiat Modarres University of Iran, Tehran, in 1996 and 2005 respectively. He works in Computer Department as an assistant professor at Ferdowsi University of Mashhad. His research interests include adaptive filtering, image and video processing, and optimization in signal processing. He has more than 140 journal and conference publications in subject of interesting area.

Email: h-sadoghi@um.ac.ir, sadoghi@sttu.ac.ir

Mojtaba Lotfizad was born in Tehran, Iran, in 1955. He received the B.S degree in electrical engineering from Amir Kabir University, Iran, in 1980, and the M.S. and Ph.D. degrees fromtheUniversity ofWales, UK, in 1985 and 1988, respectively. He then joined the Engineering Faculty Tarbiat Modarres University, Iran. He has also been a Consultant to several industrial and government organizations. His current research interests are signal processing, adaptive filtering, speech processing, and specialized processors.

Email: lotfizad@modares.ac.ir 